\section{Posterior and anterior cortex - where is the difference that makes the difference?}

\author{
Christof Koch, Marcello Massimini, Melanie Boly and Giulio Tononi
}

We thank Sandberg, Frässle and Pitts for their perceptive letter about our Review (Neural correlates of consciousness: progress and problems. Nat. Rev. Neurosci. 17, 307-321 (2016) $)^{1}$. The correspondents raise the issue of future directions (Future directions for identifying the neural correlates of consciousness. Nat. Rev. Neurosci. http://dx.doi.org/10.1038/ nrn.2016.104 (2016)) $)^{2}$, which, owing to space limitations, we addressed in the accompanying Opinion article ${ }^{3}$. In that article, we describe a principled approach - integrated information theory - that characterizes the physical substrate of consciousness as a maximum of intrinsic cause-effect power. The ensuing research strategy aims at locating such a maximum in the brain, and at determining its borders, its constituting elements, its temporal scale and its activity variables.

In the Review, we argue that it is time to consider a posterior 'hot zone', rather than a fronto-parietal network, as the likely substrate of many aspects of phenomenal experience. The notion that fronto-parietal networks may be the neural correlates of consciousness (NCC) was derived primarily from between-state and task-related contrasts that were obtained through neuroimaging studies confounded by executive control and through paradigms conflating consciousness with responsiveness. Moreover, this notion is inconsistent with the effects of extensive bilateral lesions of the prefrontal cortex, indicating that the front of the brain is not necessary for being conscious. By contrast, the importance of the posterior hot zone is highlighted by within-state, no-task paradigms that decouple experience from immediate responsiveness and report, such as studies contrasting consciousness and unconsciousness within the same state of sleep ${ }^{4}$, and by elegant no-report paradigm $\mathrm{s}^{5-7}$. This interpretation is consistent with evidence obtained from selective lesions of the posterior cortex.

Having said this, we bear no preconceived enmity to the prefrontal cortex. Indeed, searching for the NCC of specific aspects of experience (such as interoception or thought) in certain anterior regions is an important task ahead. This is why we dubbed this posterior set of regions a 'hot zone' rather than the 'full NCC. But if most anterior cortical lesions, just like cerebellar lesions, fail to affect consciousness directly, it is important to ask why. We emphasized that the unsuitability of cerebellar connectivity for information integration may be the reason why the cerebellum does not contribute to consciousness. A principled explanation of why much of the anterior cortex fails to contribute to experience, whereas much of the posterior cortex does, would be useful in singling out differences in their neural circuits that are crucial for consciousness, precisely because the anterior and the posterior cortex share so many features.

Sandberg, Frässle and Pitts rightly wonder why values of the perturbational complexity index (a transcranial magnetic stimulation (TMS)-electroencephalographic-based measure of information integration) are similar after stimulation of frontal and parietal regions, if most frontal regions do not contribute directly to consciousness. TMS is a strong, coarse-grained perturbation that activates many diverging cortical and cortico-subcortical connections; therefore, TMS of frontal targets also engages parietal circuits (see figure 2 of REF. 8). Ongoing studies are using localized, intracortical singlepulse electrical stimuli to investigate potential regional differences and hot spots of perturbational complexity. Concerning the visual awareness negativity, we agree that, although it is a promising electrophysiological correlate of experience and has posterior sources, its significance and its relation to selective attention are still unclear.

Finally, Sandberg, Frässle and Pitts suggest that the NCC should not be linked to an anatomical substrate but, perhaps, to particular computations being performed. On this, we beg to differ. As argued in our Opinion article $^{3}$, underlying consciousness is not a type of computation but a genuine physical substrate - one that must be characterized by physical manipulations and observations and that constitutes a maximum of intrinsic cause-effect power.

Christof Koch is at the Allen Institute for Brain Science, Seattle, Washington 98109, USA.

Marcello Massimini is at the Department of Biomedical and Clinical Sciences 'Luigi Sacco', University of Milan, 20157 Milan, Italy; and at the Instituto Di Ricovero e Cura a Carattere Scientifico,

Fondazione Don Carlo Gnocchi, 20148 Milan, Italy.

Melanie Boly is at the Department of Neurology and Department of Psychiatry, University of Wisconsin.

Giulio Tononi is at the Department of Psychiatry, University of Wisconsin, Madison, Wisconsin 53719, USA.

Correspondence to C.K. and G.T. christofK@alleninstitute.org; gtononi@wisc.edu

doi:10.1038/nrn.2016.105 Published online 28 Jul 2016

1. Koch, C., Massimini, M., Boly, M. \& Tononi, G. Neural correlates of consciousness: progress and problems. Nat. Rev. Neurosci. 17, 307-321 (2016).

2. Sandberg, K., Frässle, S. \& Pitts, M. Future directions for identifying the neural correlates of consciousness. Nat. Rev. Neurosci. http://dx.doi.org/10.1038/ nrn.2016.104 (2016).

3. Tononi, G., Boly, M., Massimini, M. \& Koch, C. Integrated information theory: from consciousness to its physical substrate. Nat. Rev. Neurosci. 17 , 450-461 (2016).

4. Siclari, F., LaRocque, J. J., Bernardi, G., Postle, B. R. \& Tononi, G. The neural correlates of consciousness in sleep: a no-task, within-state paradigm. Preprint at http://biorxiv.org/content/early/2014/12/30/012443 (2014).

5. Frässle, S., Sommer, J., Jansen, A., Naber, M. \& Einhäuser, W. Binocular rivalry: frontal activity relates to introspection and action but not to perception. J. Neurosci. 34, 1738-1747 (2014).

6. Pitts, M. A., Metzler, S. \& Hillyard, S. A. Isolating neural correlates of conscious perception from neural correlates of reporting one's perception Front. Psychol. 5, 1078 (2014).

7. Tsuchiya, N., Wilke, M., Frassle, S. \& Lamme, V. A No-report paradigms: extracting the true neural correlates of consciousness. Trends Cogn. Sci. 19 , 757-770 (2015)

8. Rosanova, M. et al. Natural frequencies of human corticothalamic circuits. J. Neurosci. 29, 7679-7685 (2009).

Competing interests statement

The authors declare no competing interests. 